Classification

Physics Abstracts

$06.70-73.30-82.80$

\title{
Design and implementation of a Kelvin microprobe for contact potential measurements at the submicron scale
}

\author{
Walid Nabhan $\left({ }^{1}\right)$, Alexandre Broniatowski $\left({ }^{2}\right)$, Gilles de Rosny $\left({ }^{2}\right)$ and Bernard Equer $\left({ }^{1}\right)$ \\ ( ${ }^{1}$ ) Laboratoire de Physique des Interfaces et des Couches Minces, Ecole Polytechnique, 91128 Palaiseau \\ Cedex, France \\ $\left({ }^{2}\right)$ Groupe de Physique des Solides, Universités de Paris VII et VI, 2 Place Jussieu, 75251 Paris Cedex \\ 05, France
}

(Received July 4; accepted October 26, 1994)

Résumé. - Nous présentons un instrument de mesure nouveau, alliant les techniques de microscopie en champ proche avec le principe bien connu de la sonde de Kelvin. Cet instrument effectue la mesure des variations locales du travail de sortie des électrons d'une surface par une méthode non destructive (méthode de Kelvin), et avec une précision de l'ordre de la dizaine de meV. Les performances de résolution spatiale actuellement atteintes ( 3 microns) sont assurées par l'emploi d'une sonde électrostatique gardée électriquement. Lamélioration des techniques de fabrication des sondes devrait mener sous peu à un affinement sensible de la résolution (objectif visé : $100 \mathrm{~nm}$ ). Linstrument permet par ailleurs la détection de charges statiques de surface, et se prête ainsi à une variété d'applications : mesure de la courbure des bandes à la surface des semiconducteurs et de la densité de pièges de surface dans ces matériaux ; étude de la diffusion superficielle d'impuretés dans un métal ; visualisation de domaines ferroélectriques ; étude de la triboélectricité, par exemple.

\begin{abstract}
We present a new instrument for contact potential measurements, combining the wellknown principle of the Kelvin probe (vibrating capacitor) method with the more recent developments in near-field microscopies. This instrument enables one to measure spatial variations in the electronic work function of a specimen on the submicron scale by a non-destructive technique, with a typical accuracy in the $10 \mathrm{meV}$ range. The high spatial resolution is achieved by means of an electrically guarded electrostatic probe. Developments in the probe manufacturing technique should lead in the near future to a significant improvement in the resolution, down to the $100 \mathrm{~nm}$ scale. The instrument has the additional capability of detecting static charges, hence its potential use in various fields of surface physics and technology: investigation of the band curvature at semiconductor surfaces and the measurement of surface trap densities in semiconductor devices; surface diffusion in metals; ferroelectric domain visualisation; study of triboelectricity, among many others.
\end{abstract}




\section{Introduction.}

The electronic work function stands among those materials properties most sensitive to the physical and the chemical conditions at the surface of a specimen. Thus, it is known that the surface potential of a semiconductor exhibits small scale fluctuations associated with the spatial distribution of the dopant species and with the charge state of electronic surface traps. In a different context, local work function fluctuations are of a great importance in determining the kinetics of corrosion and surface oxydation in metals. In these two examples, as in many others, there is thus a basic interest in monitoring the work function fluctuations at the local (submicron) scale. The purpose of this paper is to present a new instrument to perform such measurements, using the well-known Kelvin (vibrating capacitor) method for contact potential measurements [1] in the frame of a near-field microscope. At the present stage, the instrument allows work function variations to be measured with a $10 \mathrm{meV}$ accuracy and a spatial resolution on the micron scale. An improvement by a factor of 10 in the spatial resolution appears a realistic aim for the near future.

The principle of the method of measurement is presented in section 2 . Section 3 describes the general features of the instrument: realization of electrically guarded probes, electrical and mechanical layout and servo-controls. Experimental data demonstrating the instrument capabilities are presented in section 4 . Section 5 concludes with the prospects for future developments.

\section{Principle of the Kelvin microprobe.}

2.1 The Kelvin method For Contact potential measurements. - The Kelvin method is an intrinsically accurate and nondestructive method to measure the contact potential difference (cpd) between the surfaces of two different conducting materials. With one of the surfaces taken as the reference, this measurement provides a direct access to work function variations in the specimen under investigation. First proposed by Lord Kelvin nearly a century ago, the method has been re-examined and improved by numerous authors since [2-6].

The cpd between two surfaces is the electrostatic potential difference arising between them as they are brought into electrical contact and thermodynamic equilibrium is achieved. The principle of the Kelvin method is to set the two surfaces face to face as the plates of a capacitor $C$. An adjustable dc voltage source $V_{0}$ and a current detector are inserted in series in the circuit. The capacitor is then polarised so that it carries the charge

$$
Q=C\left(V_{0}-V_{\mathrm{k}}\right),
$$

where $V_{\mathrm{k}}$ is the cpd between the two surfaces. Assume that one of the plates is set in a sine wave motion at the frequency $f_{\mathrm{v}}$. The capacitance then varies as a function of time and so does the charge on the condenser plates. There results a current flow in the circuit, whose magnitude is given by

$$
i(t)=\frac{\mathrm{d} Q}{\mathrm{~d} t}=\frac{\mathrm{d} C}{\mathrm{~d} t}\left(V_{0}-V_{\mathrm{k}}\right)
$$

The cpd $V_{\mathrm{k}}$ is determined by ajusting $V_{0}$ so that the current vanishes. On account of the periodicity of the vibrational motion, a lock-in charge amplifier tuned to the frequency $f_{\mathrm{v}}$ can be used as the null-current detector. The measurement accuracy can then be improved to (virtually) any extent by suitably increasing the filtering time constant of the amplifier.

2.2 Simultaneous measurement of the mean SAMPle-to-PRobe Distance. - By superimposing over the d.c. voltage $V_{0}$ an a.c. signal $V_{\mathrm{c}}$ at frequency $f_{\mathrm{c}}$, an additional a.c. current is 
generated through the circuit, whose magnitude is given by

$$
i_{\mathrm{c}}(t)=2 \pi f_{\mathrm{c}} C_{0} V_{\mathrm{c}} \cos \left(2 \pi f_{\mathrm{c}} t\right)
$$

where $C_{0}$ is the zero-order term in the Fourier series expansion of $C(t) . C_{0}$ represents the average probe-sample capacitance taken over a period of the vibration $1 / f_{\mathrm{v}}$. A lock-in amplifier tuned to the frequency $f_{\mathrm{c}}$ allows thus $C_{0}$ to be determined. Assuming the capacitance-versus-distance relationship $C(z)$ to be known, this allows one to monitor the sample-to-probe distance while performing at the same time the contact potential measurement.

2.3 ORDERS OF MAGNITUDE OF THE ELECTRICAL SIGNALS AND AMPLIFIER NOISE REQUIREMENTS. - The vibrational motion generates a periodic variation in the charge of the sample-probe capacitor, whose magnitude is given by

$$
\Delta Q=\Delta C\left(V-V_{\mathrm{k}}\right)
$$

where $\Delta C$ is the amplitude of the capacitance modulation. To first order in the vibration amplitude $\Delta z$,

$$
\Delta C \approx \frac{\mathrm{d} C}{\mathrm{~d} z} \Delta z
$$

In a simple plane capacitor model, one would have $C(z)=\varepsilon_{0} S / z$ where $S$ denotes the extension area of the electrostatic field lines, whence $\Delta C=\varepsilon_{0} S\left(\Delta z / z^{2}\right)$. For a micronic lateral resolution let us take, in order of magnitude, $S \approx 5 \mu \mathrm{m}^{2}$ and $z \approx 0.1 \mu \mathrm{m}$. A typical vibration amplitude is $\Delta z \approx 30 \mathrm{~nm}$. One finds thus $\Delta C \approx 0.15 \mathrm{fF}$. Let us then denote by $\Delta f$ the measurement bandwidth about the carrier frequency $f_{\mathrm{v}} . \Delta f$ must be taken large enough that the data acquisition time remains within reasonable limits: typically $\Delta f \approx 100 \mathrm{~Hz}$. It can then be readily seen that to measure the cpd to a $10 \mathrm{mV}$ accuracy requires the charge amplifier noise to be less than $10^{-19} \mathrm{C} /(\mathrm{Hz})^{1 / 2}$. Ultra-low noise electronics is therefore a prerequisite for such an instrument. Amplifiers with suitable characteristics are available from a number of manufacturers (Amptek, Lecroy).

\section{General description of the instrument.}

3.1 Electrostatic microprobes. - In order to achieve the best possible spatial resolution, we make use of electrically guarded microprobes (Fig. 1). Such probes are made from sharply pointed needles (apex radius of curvature about $3 \mu \mathrm{m}$ ). The electrical guard is made by coating the needle with a thin layer of an insulating varnish, then metallized by aluminum evaporation. The active area of the probe is then uncovered by flashing an electrical spark between the tip and a plane metallic electrode. The spark tears off the aluminum layer on the apex of the tip. By an appropriate choice of the voltage conditions, it has been found possible to reproducibly obtain electrically guarded tips with a proper electrical insulation between core and guard, and a probe surface of a few $\mu \mathrm{m}^{2}$.

For orders of magnitude estimates, the tip can be represented as a revolution hyperboloid, facing the sample surface regarded as a plane (Fig. 2). In this figure, $R$ denotes the apex radius of curvature and $h$ is the height of the sensitive part of the probe as measured from the apex. An analytical calculation then leads to the following conclusions:

a) The probe-surface capacitance takes significant values when $z \ll R$ only. One finds in this case $C(z) \approx 1 / z$. This justifies the analysis in section 2.3 where it had been assumed that $\Delta z \ll z \ll R$. b) When $z \ll R$, the lateral extent of the field lines is given to a first approximation by $h$. 

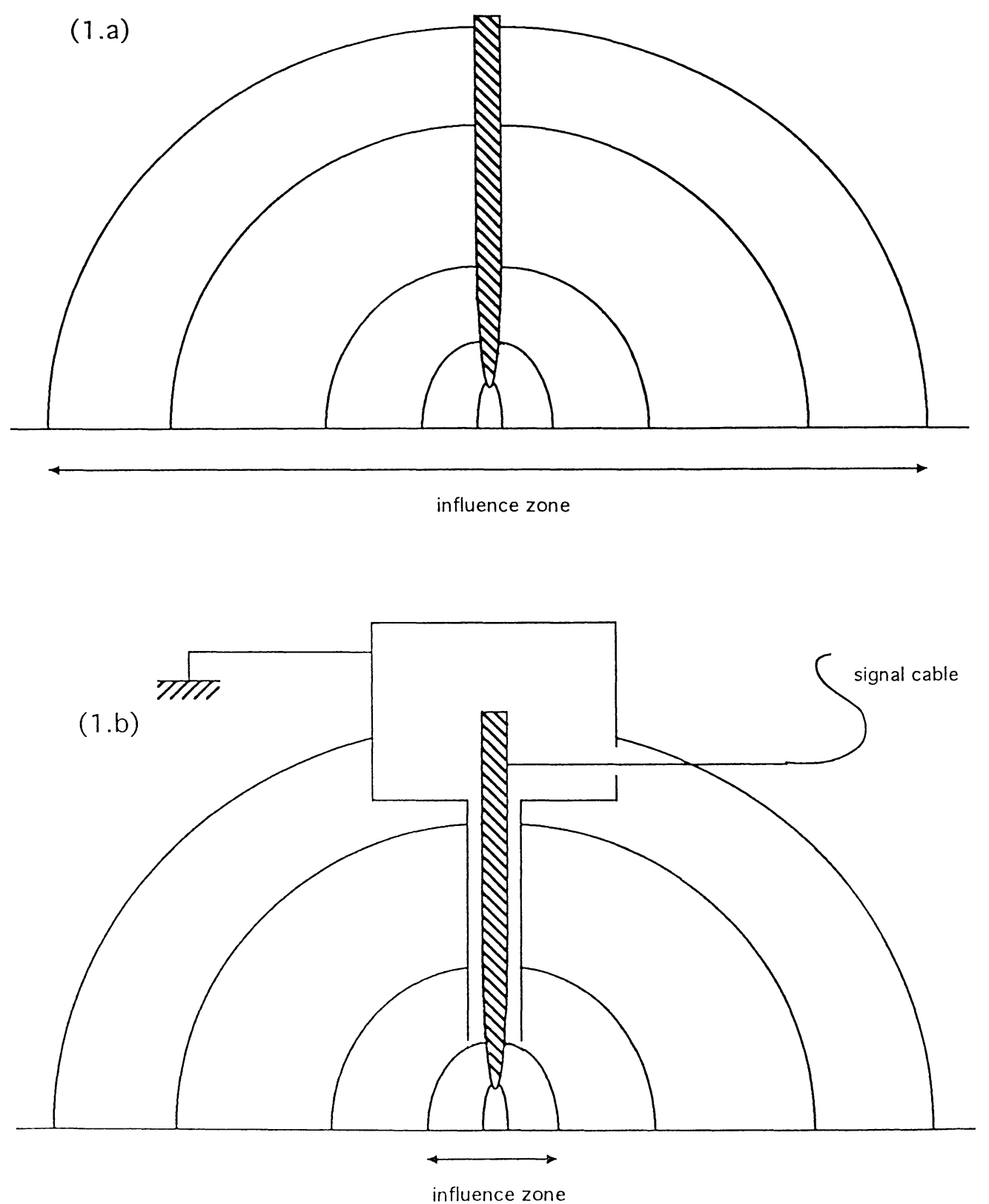

Fig. 1. - Electrostatic field lines for a plane sample and a tip-shaped probe a) without guard and b) with a grounded guard. The guard limits the extent of the probed area and provides for a better spatial resolution.

The sparking method that we use takes advantage of the enhanced electric field at the sharp tip of the probe. For an optimized procedure, we find that $h \approx R$, so that the apex radius of curvature $R$ remains as the only characteristic length of the device. Detectable electrical signals will then be obtained only when $z \ll R$, the lateral resolution of the probe then being expected to be of the order of $R$. 


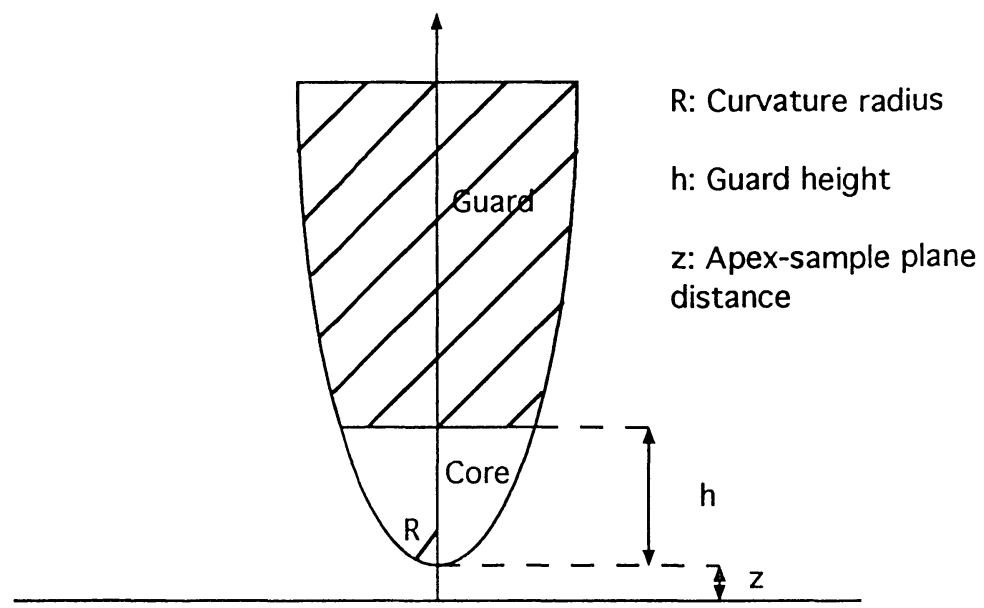

Fig. 2. - Schematic representation of the probe-tip geometry with the tip represented as a revolution hyperboloid. $R$ : curvature radius at the apex of the tip; $h$ : height of the unguarded part of the probe as measured from the apex; $z$ : specimen-apex distance.

3.2 ElECTRICAL AND MECHANICAL LAYOUT. - Figure 3 gives a schematic representation of the electrical and the mechanical layout of the instrument. The specimen is driven by an electromechanical vibrator at frequency $f_{\mathrm{v}}$ while being electrically connected to an adjustable voltage source $V=V_{0}+V_{\mathrm{c}} \cos \left(2 \pi f_{\mathrm{c}} t\right)$. The probe electrode is connected to a low noise charge amplifier whose output voltage is applied to two lock-in amplifiers in parallel, tuned at the respective frequencies $f_{\mathrm{v}}$ and $f_{\mathrm{c}}$. The probe is fixed on a piezoelectric tube, ensuring its accurate positioning by external electrical commands. The body of the instrument is supported by micrometric screws, allowing the coarse $X Y$ sample positioning as well as the $Z$ approach of the probe.

All electric cables to the probe are carefully shielded and fastened to the body of the instrument. The latter rests on an anti-vibrating stand. With these precautions taken, the limiting factor to the accuracy of the probe appears to be the internal noise of the charge amplifier. As explained before, the vibrational motion is communicated to the specimen rather than to the probe electrode. This disposition ensures that stray capacitance effects [7-8] are intrinsically minimized.

3.3 Automated operation. - The servo-control system needed for automated operation of the instrument has been carefully studied both theoretically and experimentally. A block diagram of the feedback loops is presented in figure 4.

Automated monitoring of the contact potential is achieved by returning to the specimen the output voltage from the "Kelvin" lock-in amplifier (frequency $f_{\mathrm{v}}$ ). For large enough values of the open loop gain, $V_{\mathrm{k}}$ is just given by the output voltage of the amplifier. The sample-to-probe distance is kept to the reference value by feeding the output signal of the "capacitance" lock-in amplifier (frequency $f_{\mathrm{c}}$ ) back to the $Z$ input of the piezo-electric tube, via an integrator amplifier. The purpose of the integrator is to ensure a correct response of the loop to typical perturbations.

\section{Current performances.}

Figure 5 represents a typical recording of the sample-probe capacitance as a function of distance. The test sample is made of a chromium layer, vapour-deposited on a glass substrate. An aluminum 


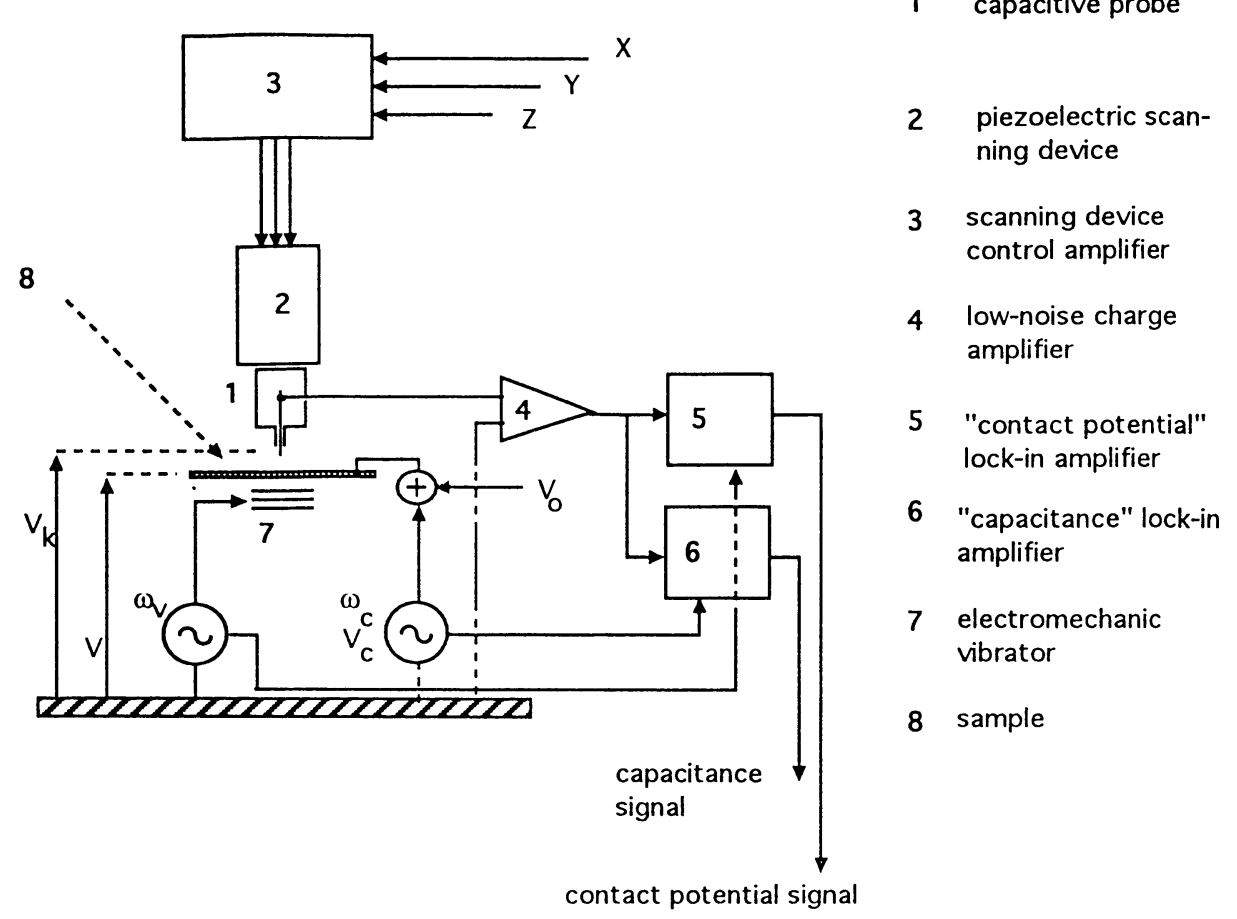

Fig. 3. - Electrical and mechanical layout of the Kelvin microprobe.

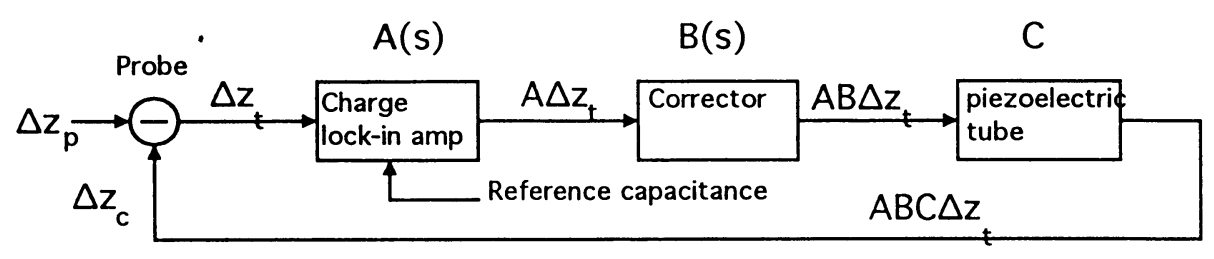

(4.a)

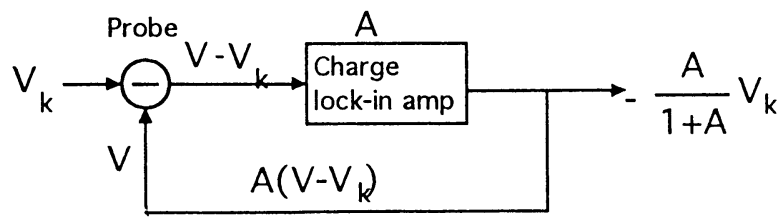

Fig. 4. - Block diagrams of the capacitance (a) and the contact potential (b) feedback loops. (a) Variations in the sample-to-tip distance arising from external perturbations and from the feedback correction yield the total variation $\Delta z_{\mathfrak{t}}$, detected as a capacitance change. The output voltage of the lock-in amplifier is returned through a signal corrector to the $Z$ input command of the piezoelectric tube. The resulting displacement $\Delta z_{\mathrm{c}}$ cancels the perturbation displacement $\Delta z_{\mathrm{p}} . \mathrm{A}(\mathrm{s}), \mathrm{B}(\mathrm{s})$ and $\mathrm{C}$ are the complex transmittances of the elements. An integrator is used as the corrector to ensure the proper response to typical perturbations. (b) The output voltage of the lock-in amplifier is returned to the specimen. This results in the output voltage being proportionnal to the contact potential $V_{\mathrm{k}}$. The correction factor $A /(1+A)$ tends to unity as the open loop gain $A$ tends to infinity. 


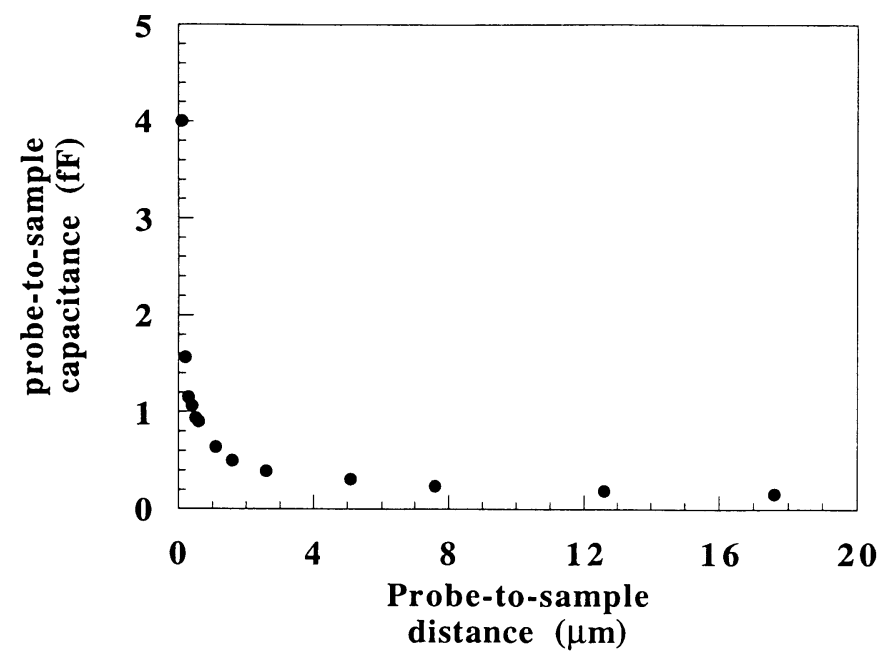

Fig. 5. - Probe-sample capacitance vs. distance.

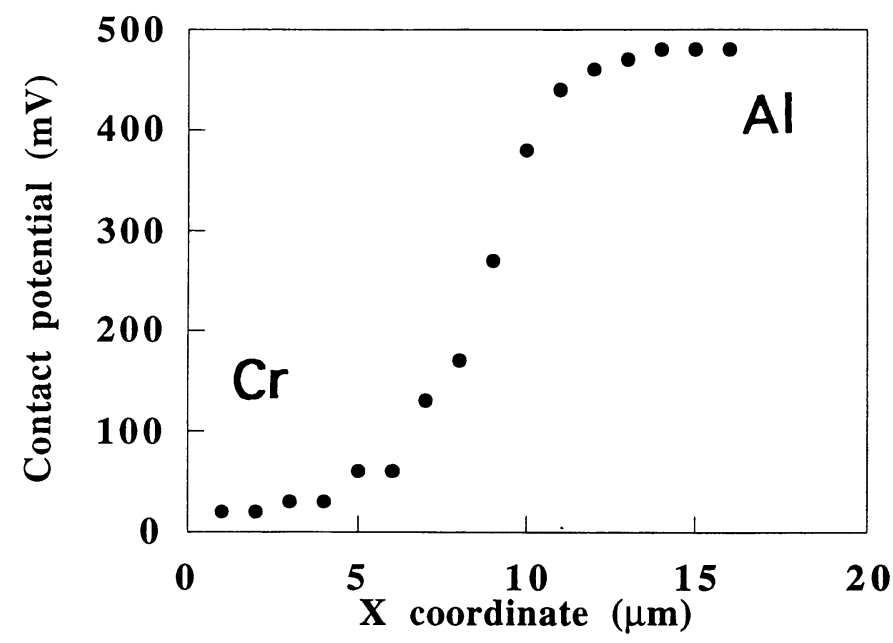

Fig. 6. - Contact potential vs. $x$ coordinate in the sample plane, showing the variation $(450 \mathrm{mV})$ in the contact potential as the tip is shifted over the edge of the aluminum layer.

overlayer about a $100 \mathrm{~nm}$ thick covers part of the chromium, thus allowing for both contact potential and thickness measurements. Figure 6 presents the data obtained for the contact potential, as the surface of the specimen was scanned manually. The contact potential exhibits a sharp variation (about $450 \mathrm{mV}$ ) as the probe is shifted over the edge of the aluminum layer. The lateral resolution (defined as the width at half maximum of the derivative of the curve in Fig. 6) is on the order of 3 micrometers.

Figure 7 represents a similar set of data with the probe operated in the automated mode. The probe displacement is now effected by means of a voltage ramp on the $X$ input of the piezoelectric tube. The line scan was performed at a fixed value of the capacitance, corresponding to a sampleto-probe distance of $0.4 \mu \mathrm{m}$. The results are similar to those obtained in the manual mode as 
(7.a)

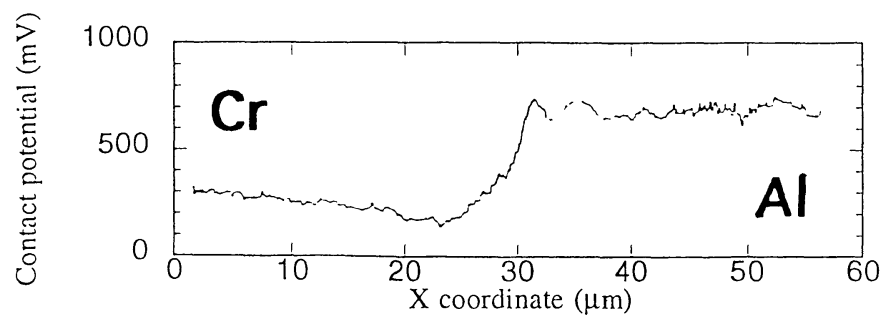

(7.b)

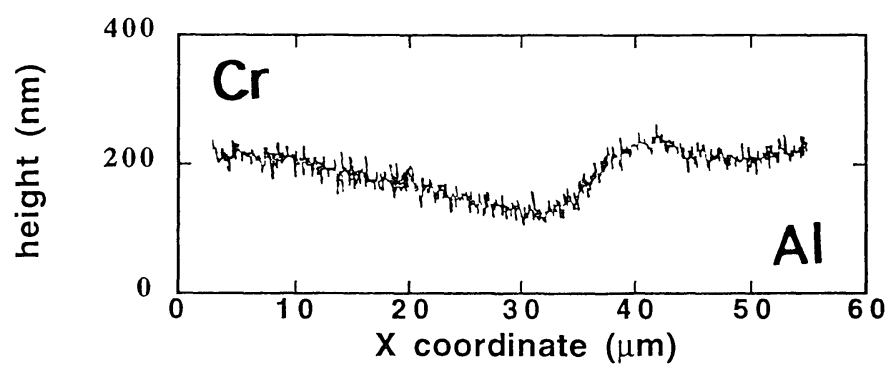

Fig. 7. - Automated line scan over the same specimen as in figure 6, showing the contact potential variation (a) and the thickness profile (b) as the tip is shifted over the edge of the aluminum layer. The slope of the sample plane with respect to the piezoelectric tube $X Y$ plane is superimposed over the thickness profile.

regards the variation of the cpd and the lateral resolution. The step in the sample thickness is also observed (Fig. 7b).

\section{Conclusion: prospects for developments.}

Besides installing a proper acquisition and imaging system, our main effort at this stage is to improve the lateral resolution of the instrument well into the submicron range. This involves basic changes in the technique of probe manufacturing, using tunnelling microscope tips with an apex radius of curvature well below $100 \mathrm{~nm}$. A technique is being currently developed for the plasma deposition on the tip of an insulating amorphous carbon layer, as this material appears to have the required qualities of high electrical resistivity, adherence and mechanical hardness [9]. A plasma etching process is also being investigated, as this is needed in the final stage of the tip preparation. The applications contemplated cover a variety of subjects, ranging from the physics of metals (surface diffusion) and semiconductors (surface states measurements) to that of insulators (triboelectricity), polymers and ferroelectric materials (domain walls). A comparison with the Kelvin probe force microscope should also prove of the highest interest [10].

\section{Acknowledgments.}

This project is supported by CNRS under contract ULTIMATECH. The authors are indebted to their colleagues in the near-field microscopy group of the Groupe de Physique des Solides for stimulating discussions and technical assistance. 


\section{References}

[1] Lord Kelvin, Philos. Mag. 46 (1898) 82.

[2] Zisman W.A., Rev. Sci. Instrum. 3 (1932) 367.

[3] Surplice N.A. and D’Arcy R.J., J. Phys. E: Sci. Instrum. 3 (1970) 477.

[4] Baikie I.D., Mackenzie S., Estrup P.J.Z. and Meyer J.A., Rev. Sci. Instrum. 62 (1991) 1326.

[5] Baumgärtner H. and Liess h.d., Rev. Sci. Instrum. 59 (1988) 802.

[6] Shelgon YI, Oriani R.A. and Stratmann M., J. Electrochem. Soc. 138 (1991) 55.

[7] Ritty B., Wachtel F., Manquenouille R. and Donnet J.B., J. Phys. E: Sci. Instrum. 15 (1982) 310.

[8] Baikie I.D., Venderbosch E., Meyer J.A. and Estrup P.J.Z., Rev. Sci. Instrum. 62 (1991) 725.

[9] "Amorphous Hydrogenated Carbon Films" in EMRS Symposium Vol. 17, Koidl and Oelhafen Eds. (Les Editions de Physique).

[10] Nonnenmacher M., O’Boyle M.P. and Wickramasynghe H.K., Appl. Phys. Lett. 58 (1991) 2921. 